\title{
PARAMETRIC PERTURBATION PROBLEMS IN ORDINARY DIFFERENTIAL EQUATIONS
}

\author{
BY
}

\author{
THOMAS G. HALLAM
}

\begin{abstract}
The asymptotic behavior of solutions of a nonlinear differential equation that arises through a nonlinear parametric perturbation of a linear system of differential equations is discussed. Fundamental hypotheses include the admissibility of a pair of Banach spaces for the linear system. Conclusions about the behavior of the perturbed system evolve through the behavior of certain manifolds of solutions of the unperturbed linear system. Asymptotic representations are found on a semi-infinite axis $R_{+}$and on the real line $R$. The bifurcation condition, which is shown to be trivial on $R_{+}$, plays an essential role for the perturbation problem on $R$. Illustrations and examples, primarily on the space $L^{\infty}$, of the theoretical results are presented.
\end{abstract}

1. Introduction. Perturbation theory of linear equations is of fundamental importance in differential equations. In this article further explorations of this area are presented; the essential features of this investigation include a linear ordinary differential equation that is perturbed parametrically by a nonlinear quantity. As is traditional in such problems, the behavior of the solutions of the perturbed equation is determined asymptotically in terms of the solutions of the linear equation.

The perturbation problem is treated here in an abstract setting. Applications of the abstract theory to specific Banach spaces are presented and form an integral portion of this paper. These results parallel and complement some of the basic literature of parametrically perturbed periodic differential systems. An excellent history of the standard works on parametric perturbations, especially those results concerned with nonlinear oscillations, may be found in the survey [4] by L. Cesari and the monograph [10] by J. K. Hale. Numerous applications as well as theoretical aspects of nonlinear oscillations may be found in [20]. Additional relevent references are contained in each of these books.

The notation of admissibility is essential in our development. The fundamental principles of admissibility for linear differential equations were promulgated and amplified by J. L. Massera and J. J. Schäffer; see, for example,

Received by the editors May 1, 1974.

AMS (MOS) subject classifications (1970). Primary 34A30, 34D10, 34E10.

Key words and phrases. Admissibility, asymptotic behavior, bounded solutions, nonlinear eigenvalue problem, parametric perturbation problem. 
the article [18] and the comprehensive compilation of their work in ordinary differential equations [19]. The book by P. Hartman [14] also contains an excellent introdyction to admissibility theory.

Results, prominent in admissibility theory, due to W. A. Coppel [6] , [7], [8] and R. Conti [5] are notable for the illustrative examples included in this article.

C. Corduneanu [9] and P. Hartman and N. Onuchic [15] have employed the concept of admissibility to develop a very general nonlinear (nonparametric) perturbation theory for ordinary differential equations. A purpose of this article is to amplify the theory of parametrically perturbed systems by employing admissibility.

Our development entails an abstract formulation of both the linear and nonlinear problems along the lines of S. Bancroft [1]; his main motivation and application were provided by periodic systems. This contrasts with our emphasis upon the admissibility concept and specific illustrations to bounded solutions. Another reference whose results will be utilized here is [13]

Differences between perturbation theorems valid on a semi-infinite axis $R_{+}=[0, \infty)$ and those valid on the entire line $R=(-\infty, \infty)$ are given special consideration in the sequel. A main distinction between these two domains is that a "bifurcation" condition which is trivial on $R_{+}$plays an important role on the interval $R$. The linear and nonlinear problems on $R_{+}$are explored in $\S \S 3$ and 4 respectively; the analogues on $R$ are found in $\S \S 5$ and 6 .

2. Preliminary notation and results. Let $J$ denote one of the intervals $R=(-\infty, \infty), R_{+}=[0, \infty)$, or $R_{-}=(-\infty, 0]$. In the differential equations

$$
d y / d t=A(t) y, \quad t \in J, y \in \mathbf{R}^{n},
$$

and

$$
d x / d t=A(t) x+f(t), \quad t \in J, x \in \mathbf{R}^{n},
$$

$A=A(t)$ is a measurable $n \times n$ matrix function that is locally integrable on $J$ and $f=f(t)$ is a measurable $n$-vector function that is locally integrable on $J$.

Let $Y(t)$ denote the fundamental matrix solution of the initial value problem $d Y / d t=A(t) Y, Y(0)=I_{n}, t \in J$.

The norm of a Banach space $\mathbf{B}_{J}$ of $n$-vector valued functions defined on the interval $J$ will be designated by $|\cdot|_{B_{J}}$. We denote by $L_{J}$ the space of locally Lebesgue integrable $R_{n}$-valued functions defined on $J$ with the topology of local convergence in the mean of order one. A Banach space $B_{J}$ is stronger than $\mathbf{L}_{J}$ if $\mathbf{B}_{\boldsymbol{J}}$ is algebraically contained in $\mathbf{L}_{J}$ and convergence in $\mathbf{B}_{J}$ implies convergence in $\mathbf{L}_{\boldsymbol{J}}$. Each Banach space employed in this article shall consist of measurable 
functions from $J$ to $\mathbf{R}^{n}$ and all such spaces will be tacitly assumed to be stronger than $\mathbf{L}_{\boldsymbol{J}}$.

A pair of Banach spaces $\left(\mathbf{B}_{J}, \mathbf{D}_{J}\right)$ is admissible for (1) if for each $f$ in $\mathbf{B}_{J}$ (2) has a solution $\boldsymbol{x}$ in $\mathbf{D}_{\boldsymbol{J}}$.

For notational convenience, a " + " or "-" subscript will indicate that the domain $J$ is $R_{+}$or $R_{-}$respectively. The absence of a subscript will imply $J=R$.

A requirement employed in $\S \S 5$ and 6 , when the domain space $J=R$, is the following. Each Banach space $\mathrm{B}=\mathrm{B}_{R}$ has the property that whenever $u$ is in B and $v$ is in $\mathbf{L}$ with $|v(t)| \leqslant|u(t)|$ for almost all $t$ in $R$ then $v$ is in B and $|v|_{\mathrm{B}} \leqslant|u|_{\mathrm{B}}$. If $u$ is in $\mathrm{L}\left(=\mathrm{L}_{R}\right)$, define $u_{+}$by $u_{+}(t)=u(t)$ if $t \geqslant 0, u_{+}(t)=0$ if $t \leqslant 0$; let $u_{-}$be given by $u_{-}(t)=u(t)$ if $t \leqslant 0$ and $u_{-}(t)=0$ if $t>0$. If $u$ is an element in a Banach space $\mathrm{B}$, then so are $u_{+}$and $u_{-}$. On the other hand, if $u_{+}$and $u_{-}$are in $\mathrm{B}$ then $u=u_{+}+u_{-}$almost everywhere and, consequently, $\boldsymbol{u}$ is in $\mathbf{B}$.

Define the set $\mathbf{B}_{+}$to consist of all functions $f$ that are in $L_{+}$and there is a member $g$ of B such that $f(t)=g(t)$ for $t$ in $R_{+}$. Let $|f|_{\mathrm{B}_{+}}=\left|g_{+}\right|_{\mathrm{B}}$; note $|\cdot|_{B_{+}}$is well defined. The distinction between elements of the component space $\mathbf{B}_{+}$of $\mathbf{B}$ and the Banach space $\mathbf{B}_{R}$ is not significant in our investigation and no effort shall be made to differentiate between such functions. In an analogous fashion the component space B_ of B can be defined.

Let $\mathbf{D}$ be a Banach space and $\mathbf{D}_{+}, \mathbf{D}_{-}$designate its component spaces. Denote by $\mathbf{M}_{\mathrm{OD}}$ the linear subspace of $\mathbf{R}^{n}$ such that $y_{0}$ is in $\mathbf{M}_{\mathrm{OD}}$ if and only if the solution $y$ of (1) with $y(0)=y_{0}$ is in $\mathbf{D}$. Let $\mathbf{M}_{-1 \mathrm{D}}$ be the linear subspace of $\mathbf{R}^{n}$ with the property that $\mathbf{M}_{\mathbf{O D}} \oplus \mathbf{M}_{-1 \mathrm{D}}$ consists of all $y_{0}$ in $\mathbf{R}^{n}$ such that the solution $y$ of (1) with $y(0)=y_{0}$ is in $\mathbf{D}_{+}$. The space $\mathbf{M}_{1 \mathrm{D}}$ is defined similarly by utilizing the component space $\mathbf{D}_{-}$; hence, $y_{0}$ is in $\mathbf{M}_{\mathbf{O D}} \oplus \mathbf{M}_{1 \mathrm{D}}$ if and only if the solution $y$ of (1) with $y(0)=y_{0}$ is in $D_{-}$. A subspace complementary to $\mathbf{M}_{\mathbf{0 D}} \oplus \mathbf{M}_{-1 \mathrm{D}} \oplus \mathbf{M}_{1 \mathbf{D}}$ will be designated by $\mathbf{M}_{\infty \mathbf{D}}$. Supplementary projections $P_{0 \mathrm{D}}, P_{-1 \mathrm{D}}, P_{1 \mathrm{D}}$, and $P_{\infty \mathrm{D}}$ can be defined on $\mathrm{R}^{n}$ to have ranges $M_{0 D}, M_{-1 D}, M_{1 D}$, and $M_{\infty D}$ respectively.

A Banach space that plays an important role in our investigations is now defined. Let $\mathbf{B}_{J}$ and $\mathbf{D}_{J}$ be Banach spaces and $L: \mathbf{D}_{J} \rightarrow \mathbf{B}_{J}$ be the operator defined by $L y=y^{\prime}-A(t) y$. The space $\mathrm{E}_{J}=\mathrm{E}_{J}(\mathrm{D}, \mathrm{B})=\left\{y: y \in \mathrm{D}_{J}, L y \in \mathrm{B}_{J}\right\}$ is a Banach space with norm $|y|_{E_{J}}=|y|_{D_{J}}+|L y|_{B_{J}}$ (see [22, p. 196]).

If the pair of Banach spaces $\left(\mathrm{B}_{+}, \mathrm{D}_{+}\right)$is admissible for (1) where $J=R_{+}$, then for prescribed $f$ in $\mathbf{B}_{+}$, there is exactly one solution $x$ of (2) in $D_{+}$satisfying the condition $\left(P_{0 T}+P_{-1 T}\right) x(0)=0$.

It is a consequence of the above fact that the linear function $T_{+}: \mathbf{B}_{+} \rightarrow$ $\mathbf{D}_{+}$, given by $T_{+} f=x$, where $f$ is in $\mathbf{B}_{+}$and $x$ is the unique solution of (2) in 
$D_{+}$satisfying $\left(P_{0 \mathrm{D}}+P_{-1 \mathrm{D}}\right) x(0)=0$, is well defined. In a similar manner, the operator $T_{-}: \mathrm{B}_{-} \rightarrow \mathrm{D}_{-}$is defined for $t$ in the interval $R_{-}$.

It follows from [18, p. 295] that there exists a positive constant $K$ (depending only upon $A$ and not upon $f$ ) such that $\left|T_{ \pm} f\right|_{\mathbf{D}_{ \pm}} \leqslant K|f|_{\mathbf{B}_{ \pm}}$. This implies that the operators $T_{ \pm}$are continuous from $B_{ \pm}$to $D_{ \pm}$.

3. Abstract formulation of the linear problem on $R_{+}$. A basic assumption made about equation (1) in this section is

H.3: The pair of Banach spaces $\left(B_{+}, D_{+}\right)$is admissible for (1). If (H.3) is satisfied, then the pair of Banach spaces $\left(\mathbf{B}_{+}, \mathbf{E}_{+}\right)$is also admissible for (1) $\left(E_{+}\right.$is defined in $\left.\S 2\right)$. Our first result analyzes the linear operator $L$ : $\mathbf{E}_{+} \rightarrow \mathbf{B}_{+}$defined by $L y(t)=d y(t) / d t-A(t) y(t)$.

THEOREM 3.1. Suppose that (H.3) is satisfied. The operator $L: \mathbf{E}_{+} \rightarrow$ $\mathbf{B}_{+}$is a closed linear operator whose nullity $\mathbf{N}(L)$ is a closed subset of $\mathbf{E}_{+}$admitting projection in $\mathbf{E}_{+}$; that is, there exists a projection $\Pi_{+}: \mathbf{E}_{+} \rightarrow \mathbf{E}_{+}$such that $\mathbf{N}(L)=\mathbf{R}\left(\Pi_{+}\right)$. The range $\mathbf{R}(L)$ is all of $\mathbf{B}_{+}$. Furthermore, $L$ has a bounded right inverse $K: \mathrm{B}_{+} \rightarrow \mathrm{E}_{+}$; that is $L K=I_{\mathrm{B}_{+}}$.

$P_{R O O F}$. It was assumed that each of the Banach spaces $\mathbf{B}_{+}$and $\mathbf{D}_{+}$are stronger than $\mathbf{L}_{+}$. The fact that $L$ is closed is contained in the following lemma of Massera and Schäffer [18, p.289]:

Let $\left\{f_{n}\right\}$ be a sequence of functions in $\mathrm{L}_{+}$and let $x_{n}$ be a solution of $d x_{n} / d t=A(t) x_{n}+f_{n}(t)$ for each $n$. If the limits $\lim _{n \rightarrow \infty} f_{n}=f$ and $\lim _{n \rightarrow \infty} x_{n}$ $=x$ both exist (in $\mathrm{L}_{+}$), then the function $x$ is (except for equivalence almost everywhere) $a$ solution of (2) and $x_{n} \rightarrow x$ uniformly on every bounded subinterval of $R_{+}$as $n \rightarrow \infty$.

To demonstrate that $\mathrm{N}(L)$ is a closed subspace of $\mathrm{E}_{+}$, observe that $y$ is in $\mathrm{N}(L)$ if and only if $y$ is in $\mathrm{E}_{+}$and $y(\cdot)=Y(\cdot) \xi$ for some $\xi$ in $R^{n}$. Using the projections $P_{i \mathrm{D}}, i=0, \pm 1, \infty, Y(\cdot) \xi$ can be written as

$$
Y(\cdot) \xi=Y(\cdot)\left(P_{0 \mathrm{D}}+P_{-1 \mathrm{D}}\right) \xi+Y(\cdot)\left(P_{1 \mathrm{D}}+P_{\infty \mathrm{D}}\right) \xi .
$$

It follows from the definition of these projections that $\mathrm{N}(L)=\{y: y(\cdot)=$ $\left.Y(\cdot) \xi,\left(P_{1 \mathrm{D}}+P_{\mathrm{\infty D}}\right) \xi=0\right\}$; hence, $\mathrm{N}(L)$ is a finite-dimensional subspace of $\mathbf{E}_{+}$. The existence of the projection $\Pi_{+}$can now be obtained since every finitedimensional subspace of a Banach space is closed and admits projection.

Next, we show that $\mathbf{R}(L)=\mathbf{B}_{+}$. Let $f$ be in $\mathbf{B}_{+}$; if $\xi$ is in $R^{n}$ and $T_{+} f$ is as defined in $\S 2$, then the function $x(\cdot)=Y(\cdot)\left(P_{0 \mathrm{D}}+P_{-1 \mathrm{D}}\right) \xi+T_{+} f(\cdot)$ is a solution of $L y=f$. Thus, $\mathrm{R}(L)=\mathrm{B}_{+}$.

For theoretical purposes, it is sufficient to establish the existence of a 
bounded right inverse $K: \mathbf{B}_{+} \rightarrow \mathbf{E}_{+}$of the operator $L$. This may be accomplished through an application of Lemma 4, p. 72, of [21].

However, to be slightly more constructive, we note that $K=T_{+}$is continuous from $B_{+}$to $E_{+}$and $L T_{+}=I_{B_{+}}$. This completes the proof of Theorem 3.1.

Our next result provides a concrete illustration of Theorem 3.1 in the case $\left(\mathrm{B}_{+}, \mathrm{D}_{+}\right)=\left(\mathrm{L}^{p}\left(R_{+}\right), L^{\infty}\left(R_{+}\right)\right), 1 \leqslant p \leqslant \infty$. The hypothesis $\mathrm{H} .3$ employed in the previous theorem takes the form

H.3p. Let $q$ satisfy $1 / p+1 / q=1$ and let there exist a positive constant $K$ such that

$$
\begin{aligned}
& {\left[\int_{0}^{t}\left|Y(t)\left(P_{0 L^{\infty}}+P_{-1 L^{\infty}}\right) Y^{-1}(s)\right|^{q} d s\right]^{1 / q}} \\
& \quad+\left[\int_{t}^{\infty}\left|Y(t)\left(P_{1 L^{\infty}}+P_{\infty L^{\infty}}\right) Y^{-1}(s)\right|^{q} d s\right]^{1 / q} \leqslant K, \quad t \in R_{+} .
\end{aligned}
$$

Condition H.3p is necessary and sufficient for the pair $\left(\mathbf{L}^{p}\left(R_{+}\right), \mathbf{L}^{\infty}\left(R_{+}\right)\right)$to be admissible for (1). This result is due to Coppel [6], [8] for $p=1$ and Conti [5] in the case $1<p<\infty$.

Theorem 3.1, with $\mathbf{E}_{+}$taken as $\mathbf{E}_{+, \infty, p}=\left\{y: y \in \mathrm{L}^{\infty}\left(R_{+}\right), L y \in \mathrm{L}^{p}\left(R_{+}\right)\right\}$, implies the existence of a projection $\Pi_{+}$with the property $\mathrm{N}(L)=R\left(\Pi_{+}\right)$and that the bounded right inverse $K$ of $L$ is $T_{+}$. For computational purposes, the representations of $\Pi_{+}$and $T_{+}$are important; these representations are the contribution of the next theorem.

THEOREM 3.2. Let condition H.3p be satisfied.

(i) Suppose that $\Phi(\cdot)$ denotes the $n \times r$ matrix-valued function whose columns form a basis for $\operatorname{sp}\left\{Y(\cdot)\left(P{ }_{0 L^{\infty}}+P_{-11}^{\infty}\right)\right\}$. Then, the $r \times r$ matrix $C \equiv \int_{0}^{\infty} \Phi^{T}(s) \Phi(s) d s$ is well defined and nonsingular provided $P_{0 \mathrm{~L}}{ }^{\infty}+P_{-1 \mathrm{~L}^{\infty}}$ $\neq 0\left(\Phi^{T}\right.$ denotes $\Phi$ transpose $)$. Furthermore, the map $\Pi_{+}: \mathbf{E}_{+, \infty, p} \rightarrow \mathbf{E}_{+, \infty, p}^{-1 \mathbf{L}}$ given by

$$
\Pi_{+} \phi(\cdot)=\Phi(\cdot) C^{-1} \int_{0}^{\infty} \Phi^{T}(s) \phi(s) d s
$$

is a projection of $\mathbf{E}_{+, \infty, p}$ into $\mathbf{E}_{+, \infty, p}$ with $\mathbf{N}(L)=\mathbf{R}\left(\Pi_{+}\right)$.

(ii) $A$ bounded right inverse $K: \mathbf{L}^{p}\left(R_{+}\right) \rightarrow \mathbf{E}_{+, \infty, p}$ for $L: \mathbf{E}_{+, \infty, p} \rightarrow$ $L^{p}\left(R_{+}\right)$is given by

$$
\begin{aligned}
T_{+} f(t)= & \int_{0}^{t} Y(t)\left(P_{0 L^{\infty}}+P_{-1 \mathrm{~L}}^{\infty}\right) Y^{-1}(s) f(s) d s \\
& -\int_{t}^{\infty} Y(t)\left(P_{1 \mathrm{~L}}+P_{\infty \mathrm{L}}^{\infty}\right) Y^{-1}(s) f(s) d s, \quad t \in R_{+} .
\end{aligned}
$$

Proof. First, (i) will be established. There exist positive constants $N$ and $\alpha$ such that 


$$
|\Phi(t)| \leqslant N e^{-\alpha t}, \quad t \in R_{+}
$$

(see $[8$, p. 68] for $p=1,[12$, p. 359] for $1 \leqslant p \leqslant \infty$ ). Employing the estimate of (4), we observe that the matrix $C$ is well defined.

To show that $C$ is nonsingular, suppose that $C a=0, a$ in $R^{r}$. It follows that $\int_{0}^{\infty}|\Phi(s) a|^{2} d s=0$; hence, $|\Phi(t) a|=0$ which yields $a=0$.

The inequality (4) implies that $\int_{0}^{\infty} \Phi^{T}(s) \phi(s) d s$ is well defined for each $\phi$ in $\mathbf{E}_{+, \infty, p} \subset \mathbf{L}^{\infty}\left(R_{+}\right)$; hence, $\Pi_{+}: \mathbf{E}_{+, \infty, p} \rightarrow \mathbf{E}_{+, \infty, p}$ is also well defined. Finally, it is evident that $R\left(\Pi_{+}\right)=\left\{\phi: \phi(\cdot)=\Phi(\cdot) a_{0}, a_{0} \in R^{r}\right\}$ and that $\Pi_{+}$ is idempotent on its range; that is, $\Pi_{+}^{2} \Phi=\Pi_{+} \Phi=\Phi$. Thus, $\Pi_{+}$is a projection operator whose range is $\mathrm{N}(L)$.

To verify (ii), we note that hypothesis H.3p coupled with Hölder's inequality shows that $T_{+}$maps $L^{p}\left(R_{+}\right)$into $\mathbf{E}_{+, \infty, p}$. A direct computation shows that $L T_{+} f=f$ for $f$ in $L^{p}\left(R_{+}\right)$. This completes the proof of Theorem 3.2.

REMARK. The operator $T_{+}$contained in Theorem 3.2 has been widely used in connection with perturbation problems; see [3], [5], [8], [12]. The dual of Theorem 3.2 for periodic systems can be found in Hale [11, p. 263] .

4. Abstract formulation of the nonlinear problem on $R_{+}$. In this section, a treatment of nonlinear parameter perturbation problems on a semi-infinite axis is presented. Let $\Lambda, \mathbf{B}_{+}$, and $\mathbf{D}_{+}$denote Banach spaces and suppose that the function $N: \mathrm{D}_{+} \times \Lambda \rightarrow \mathrm{B}_{+}$and its Fréchet derivative $\partial N / \partial \phi$ are continuous on $\mathrm{D}_{+} \times \Lambda$. The sphere of radius $\rho$ in a Banach space $\mathrm{C}$ will be represented by $S_{\rho}(\mathrm{C})$. The function $N$ is weakly nonlinear over the sphere $S_{\rho}\left(\mathrm{D}_{+}\right)$if there exists a contiruous function $\eta(\rho, \sigma)$ with the properties $\eta(\rho, 0)=0$ and

$$
|N(\cdot, \lambda)|_{S_{\rho}\left(\mathbf{B}_{+}\right)} \leqslant \eta(\rho, \sigma)
$$

for all $\lambda \in S_{\sigma}(\Lambda)$. In (5), the norm $|\cdot|_{S_{\rho}\left(B_{+}\right)}$is defined by

$$
|N(\cdot, \lambda)|_{S_{\rho}\left(\mathbf{B}_{+}\right)} \equiv \sup _{\phi \in S_{\rho}\left(\mathrm{D}_{+}\right)}\left\{|N(\phi, \lambda)|_{\mathbf{B}_{+}}+\left|\frac{\partial N}{\partial \phi}(\phi, \lambda)\right|_{\mathbf{B}_{+}}\right\} .
$$

The function $N$ is a weak nonlinearity if $N$ is weakly nonlinear over every sphere $S_{\rho}\left(D_{+}\right)$in $D_{+}, \rho>0$.

The notation of the previous section is employed in the next result.

THEOREM 4.1. Let the pair $\left(B_{+}, D_{+}\right)$of Banach spaces be admissible for equation (1) and suppose that $\mathrm{E}_{+}$is the Banach space defined in $\$ 2$. Let $N: \mathbf{E}_{+} \times \Lambda \rightarrow \mathbf{B}_{+}$be a weak nonlinearity. Suppose that $M=|\Phi(\cdot)|_{\mathbf{D}_{+}}$, where $\Phi(\cdot)$ is the $n \times r$ matrix function whose columns form a basis for $\mathrm{sp}\left\{Y(\cdot)\left(P_{\mathrm{OD}}+P_{-1 \mathrm{D}}\right)\right\}$.

Then, corresponding to each $\rho>0$, there exists $a \sigma=\sigma(\rho)>0$ such that the problem 


$$
L \phi=N(\phi, \lambda), \quad \Pi_{+} \phi=\Phi(\cdot) a_{0}, \quad \phi \in S_{\rho}\left(\mathrm{E}_{+}\right),
$$

has a unique solution $\phi^{*}\left(\cdot, a_{0}, \lambda\right)$ for each $\left(a_{0}, \lambda\right) \in S_{\rho / 2 m}\left(R^{r}\right) \times S_{\sigma}(\Lambda)$.

Furthermore, $\phi^{*}\left(\cdot, a_{0}, \lambda\right)$ is continuous as a map from $S_{\rho / 2 m}\left(\mathbf{R}^{r}\right) \times S_{\sigma}(\Lambda)$ into $\mathbf{E}_{+}$and satisfies $\lim _{\lambda \rightarrow 0} \phi^{*}\left(\cdot, a_{0}, \lambda\right)=\Phi(\cdot) a_{0}$ for every $a_{0}$ in $S_{\rho / 2 m}\left(\mathbf{R}^{\eta}\right)$.

Proof. A solution to the problem (6) can be found by solving the alternate problem

$$
\phi=\left(I-\Pi_{+}\right) K N(\phi, \lambda)+\Phi(\cdot) a_{0}, \quad \phi \in S_{\rho}\left(\mathrm{E}_{+}\right) .
$$

Let the operator $T: \mathbf{E}_{+} \times \mathbf{R}^{r} \times \Lambda \rightarrow \mathbf{E}_{+}$be defined by

$$
T\left(\phi, a_{0}, \lambda\right)=\left(I-\Pi_{+}\right) K N(\phi, \lambda)+\Phi(\cdot) a_{0} .
$$

Utilizing the hypothesis that $N$ is a weak nonlinearity, the mean value theorem for Fréchet differentials [16, Corollary 1.6.2, p. 20], and some direct computation, it can be shown that $T\left(\cdot, a_{0}, \lambda\right)$ is uniformly contracting $[11, \mathrm{p} .6]$ on the set $\left\{\phi: \phi \in S_{\rho}\left(\mathrm{E}_{+}\right), \Pi_{+} \phi \in S_{\rho / 2}\left(\mathrm{E}_{+}\right)\right\}$with respect to the parameter set $S_{\rho / 2 m}\left(R^{\prime}\right) \times S_{\sigma}(\Lambda)$ provided $\sigma$ is sufficiently small.

The unique fixed point, $\phi^{*}$, of this contraction mapping inherits its continuity properties with respect to the parameters $\left(a_{0}, \lambda\right)$ from the smoothness of the uniform contraction map $T\left(\cdot, a_{0}, \lambda\right)[11, \mathrm{p} .7$, Theorem 3.2]. It is evident from the definition of $T$ together with the preceding remark that

$$
\lim _{\lambda \rightarrow 0} \phi^{*}\left(\cdot, a_{0}, \lambda\right)=\Phi(\cdot) a_{0} .
$$

This completes the proof of Theorem 4.1.

REMARK 4.1. Theorem 4.1 allows perturbation terms that are weak nonlinearities. For more general types of nonlinear perturbations, a convenient tool that may be used to replace the uniform contraction mapping principle is the Schauder-Tychonoff fixed point theorem. Perturbation results for the parameter free problem that employ this theorem in conjunction with a general admissibility requirement may be found in the article [15] by P. Hartman and $\mathrm{N}$. Onuchic. In [2], the Schauder-Tychonoff theorem was utilized in conjunction with the admissibility of the pair $\left(L^{p}, L^{\infty}\right)$ to treat a parametric perturbation problem. The next theorem extends the results of [2] to a general admissibility setting.

As noted in [15], it is convenient to use instead of the topology of $\mathbf{L}_{+}$ that of $\mathbf{C}_{+}\left(\mathbf{R}^{n}\right)$, the space of continuous functions from $R_{+}$to $\mathbf{R}^{n}$ with the compact-open topology. Let $\Sigma_{+}=S_{\rho}\left(\mathbf{E}_{+}\right) \cap \mathbf{C}_{+}\left(\mathbf{R}^{n}\right)$ and $\bar{\Sigma}_{+}$represent the closure of $\boldsymbol{\Sigma}_{+}$in $\mathbf{C}_{+}\left(\mathbf{R}^{n}\right)$.

THEOREM 4.2. Let the following assumptions be satisfied for $\rho>0$.

(i) $\left(\mathbf{B}_{+}, \mathbf{D}_{+}\right)$is admissible for (1) and $\mathbf{E}_{+}$is as defined in $\$ 2$.

(ii) The function $G$ : $\bar{\Sigma}_{+} \times \Lambda \rightarrow \mathrm{B}_{+}$defined by $G(x, \lambda)=g(\cdot, x(\cdot), \lambda)$ is continuous. 
(iii) There exists a continuous function $r: \Lambda \rightarrow R$ with $\lim _{\lambda \rightarrow 0} r(\lambda)=0$ such that

$$
|g(\cdot, x(\cdot), \lambda)|_{\mathbf{B}_{+}} \leqslant r(\lambda) \text { for all } x(\cdot) \text { in } \bar{\Sigma}_{+}, \lambda \in \Lambda \text {. }
$$

(iv) There exists a function $\gamma: R_{+} \times \Lambda \rightarrow \mathrm{L}_{+}$such that

$$
|g(t, x(t), \lambda)| \leqslant \gamma(t, \lambda) \text { for all } x(\cdot) \in \bar{\Sigma}_{+}, \lambda \in \Lambda \text {. }
$$

Then, for the prescribed $\rho$, there exists $a \sigma=\sigma(\rho)>0$ such that

$$
d x / d t=A(t) x+g(t, x, \lambda)
$$

has a family, $x^{*}\left(\cdot, a_{0}, \lambda\right)$, of solutions with the properties $x^{*}\left(\cdot, a_{0}, \lambda\right)$ is in $S_{\rho}\left(\mathbf{E}_{+}\right)$and $\lim _{\lambda \rightarrow 0} x^{*}\left(\cdot, a_{0}, \lambda\right)=\Phi(\cdot) a_{0}$ for each $\left(a_{0}, \lambda\right)$ in $S_{\rho / 2 M}\left(\mathbf{R}^{\eta}\right) \times S_{\sigma}(\Lambda)$.

Proof. The details of the proof are very similar to those of [15] and only a brief outline is presented here. As we saw in Theorem 4.1., an essential portion of the proof was to demonstrate that the operator $T: \bar{\Sigma}_{+} \times \mathbf{R}^{r} \times \Lambda \rightarrow \mathbf{E}_{+}$ given by

$$
T\left(\phi, a_{0}, \lambda\right)=\left(I-\Pi_{+}\right) K g(\cdot, \phi, \lambda)+\Phi(\cdot) a_{0}
$$

has a fixed point $\phi$ in $\mathbf{E}_{+}$. In the present situation this is accomplished by the Schauder-Tychonoff fixed point theorem. The continuity of $T$ is demonstrated much like that in $[15$, p. 1195]. The fact that $T \bar{\Sigma}[\subset \bar{\Sigma}]$ has a compact closure follows from Ascoli's Theorem; this is a main reason to employ the topology of $C_{+}$rather than that of $L_{+}$. The fixed point $x^{*}$ of $T$ in $S_{\rho}\left(E_{+}\right)$satisfies the equation

$$
x^{*}\left(\cdot, a_{0}, \lambda\right)=\left(I-\Pi_{+}\right) K g\left(t, x^{*}\left(t, a_{0}, \lambda\right), \lambda\right)+\Phi(\cdot) a_{0} .
$$

From this hypothesis (iii), we obtain the estimate

$$
\left|x^{*}\left(\cdot, a_{0}, \lambda\right)-\Phi(\cdot) a_{0}\right| \leqslant r(\lambda)
$$

from which it follows that $\lim _{\lambda \rightarrow 0} x^{*}\left(\cdot, a_{0}, \lambda\right)=\Phi(\cdot) a_{0}$.

REMARK 4.2. The previous results in this section have imposed various smoothness requirements upon the allowable perturbations then the growth rates of the solutions were discussed. The remainder of the section is devoted to power series expansion for the solutions $x^{*}$ whose existence was demonstrated in Theorem 4.2.

For a historical starting point, the survey of Cesari [4] indicates the evolution of series expansions of periodic solutions of periodic systems. The elimination of secular terms in such expressions was a difficulty that was achieved through methods similar to those used here. 
The purpose of the subsequent remarks is to develop a technique that, under hypotheses additional to those of Theorem 4.1, exhibits an expansion for the solution $x^{*}$ (in the space $\mathbf{E}_{+}$) in powers of $\lambda$ whose coefficients are also in $\mathbf{D}_{+}$.

For simplicity in computation, we shall restrict our considerations to a scalar parameter $\lambda$. We shall proceed formally, assuming that $g$ has as many continuous derivatives as required for the calculations.

The objective is a series expansion of the $\mathrm{D}_{+}$solution $x^{*}\left(\cdot, a_{0}, \lambda\right)$ of $(7)$ in the form

$$
x_{\lambda}^{*}\left(t, a_{0}, \lambda\right)=x^{*}\left(t, a_{0}, 0\right)+x_{\lambda}^{*}\left(t, a_{0}, 0\right) \lambda+x_{\lambda \lambda}^{*}\left(t, a_{0}, 0\right) \lambda^{2} / 2 !+\cdots
$$

Under suitable hypotheses $x_{\lambda}^{*} \ldots \lambda\left(\cdot, a_{0}, 0\right)$ is in $\mathrm{D}_{+} ;$this need not follow from the known fact that $x^{*}\left(\cdot, a_{0}, \lambda\right)$ is in $\mathbf{D}_{+}$. First, we observe that $x^{*}\left(\cdot, a_{0}, 0\right)$; is in $\mathrm{D}_{+}$since $x^{*}\left(\cdot, a_{0}, \lambda\right)$ is in $\mathrm{D}_{+}$.

Next, we consider $x_{\lambda}^{*}\left(\cdot, a_{0}, 0\right)$; from (7), it follows that

$$
d\left[x_{\lambda}^{*}\left(t, a_{0}, 0\right)\right] / d t=A(t) x_{\lambda}^{*}\left(t, a_{0}, 0\right)+g_{\lambda}\left(t, \Phi(t) a_{0}, 0\right) .
$$

The determining equation for $x^{*}$ is obtained in the proof of Theorem 4.1 as

$$
x^{*}\left(\cdot, a_{0}, \lambda\right)=\left[I-\Pi_{+}\right] T_{+} g\left(t, x^{*}\left(t, a_{0}, \lambda\right), \lambda\right)[\cdot]+\Phi(\cdot) a_{0} .
$$

From this representation, we have

$$
x_{\lambda}^{*}\left(0, a_{0}, 0\right)=\left[I-\Pi_{+}\right] T_{+} g_{\lambda}\left(t, \Phi(t) a_{0}, 0\right)[0] ;
$$

in finding (9), we employed various continuity properties of $g_{\lambda}$ and the fact that $g_{x}(\cdot, \cdot, 0)=0$.

Hence, the vector $x_{\lambda}^{*}\left(\cdot, a_{0}, 0\right)$ is a solution of the system

$$
d z / d t=A(t) z+g\left(t, \Phi(t) a_{0}, 0\right)
$$

with initial value $x_{\lambda}^{*}\left(0, a_{0}, 0\right)$ given by $(9)$. It follows that

$$
\begin{aligned}
\left(P_{1}+P_{\infty}\right) x_{\lambda}^{*}\left(0, a_{0}, 0\right) & =\left(P_{1}+P_{\infty}\right)\left(I-\Pi_{+}\right) T_{+} g_{\lambda}\left(t, \Phi(t) a_{0}, 0\right)[0] \\
& =\left(P_{1}+P_{\infty}\right) T_{+} g_{\lambda}\left(t, \Phi(t) a_{0}, 0\right)[0] .
\end{aligned}
$$

The next stated result of D. L. Lovelady and the author [13] is helpful at this stage. Let $\left(\mathbf{B}_{+}, \mathbf{D}_{+}\right)$be admissible for (1). If $f_{+}$is in $\mathbf{B}_{+}$and $u$ is a solution of (2), then $u$ is in $\mathbf{D}_{+}$if and only if $\left(P_{1}+P_{\infty}\right) u(0)=T_{+}\left[f_{+}\right][0]$. As an immediate consequence of this, it follows that $x_{\lambda}^{*}\left(\cdot, a_{0}, 0\right)$ is in $\mathbf{D}_{+}$provided $g_{\lambda}\left(\cdot, \Phi(\cdot) a_{0}, 0\right)$ is in $\mathbf{B}_{+}$.

We continue the procedure by determining the behavior of $x_{\lambda \lambda}^{*}\left(\cdot, a_{0}, 0\right)$. A computation shows that $x_{\lambda \lambda}^{*}\left(\cdot, a_{0}, 0\right)$ is a solution of the nonhomogeneous linear equation 


$$
d z / d t=A(t) z=g_{\lambda \lambda}\left(t, \Phi(t) a_{0}, 0\right)+2 g_{x \lambda}\left(t, \Phi(t) a_{0}, 0\right) x_{\lambda}^{*}\left(t, a_{0}, 0\right)
$$

since $g_{x x}(\cdot, \cdot, 0)=g_{x}(\cdot, \cdot, 0)=0$.

Employing the initial data obtained from equation (8) we have

$$
\begin{aligned}
& x_{\lambda \lambda}^{*}\left(0, a_{0}, 0\right) \\
& \quad=\left(I-\Pi_{+}\right) T_{+}\left[g_{\lambda \lambda}\left(t, \Phi(t) a_{0}, 0\right)+2 g_{x \lambda}\left(t, \Phi(t) a_{0}, 0\right) x_{\lambda}^{*}\left(t, a_{0}, 0\right)\right][0] .
\end{aligned}
$$

Consequently,

$$
\begin{aligned}
\left(P_{1}\right. & \left.+P_{\infty}\right) x_{\lambda \lambda}^{*}\left(0, a_{0}, 0\right) \\
& =\left(P_{1}+P_{\infty}\right) T_{+}\left[g_{\lambda \lambda}\left(t, \Phi(t) a_{0}, 0\right)+2 g_{x \lambda}\left(t, \Phi(t) a_{0}, 0\right) x_{\lambda}^{*}\left(t, a_{0}, 0\right)\right][0] .
\end{aligned}
$$

Again utilizing the above result of Hallam and Lovelady, we have $x_{\lambda \lambda}^{*}\left(\cdot, a_{0}, 0\right)$ is in $\mathbf{D}_{+}$provided

$$
g_{\lambda \lambda}\left(\cdot, \Phi(\cdot) a_{0}, 0\right)+2 g_{x \lambda}\left(\cdot, \Phi(\cdot) a_{0}, 0\right) x_{\lambda}^{*}\left(\cdot, a_{0}, 0\right)
$$

is in $\mathbf{B}_{+}$.

Proceeding analogously, the coefficients of the higher order terms can be shown to be in $\mathbf{D}_{+}$.

It is interesting to observe for periodic systems that the auxiliary requirements (e.g. $g_{\lambda}\left(\cdot, \Phi(\cdot) a_{0}, 0\right)$ in $\left.\mathbf{B}_{+}\right)$follow from the original periodic hypotheses of the function $g$ : hence, no additional hypotheses are necessary to find periodic expansions of solutions of periodic systems once the representation for $T_{+}$is known.

5. Abstract formulation of the linear problem on $R$. In $\S 3$ an abstract formulation of the linear problem on a semiaxis $R_{+}$was given; $\S 4$ utilized these linear results to develop a nonlinear perturbation theory. We now explore analogues of these results on the real line $R$.

If we assume that $(B, D)$ is admissible for (1), then only notational changes in the previous results are required. A more interesting problem arises if we suppose that each of $\left(B_{+}, D_{+}\right)$and (B_, $\left.D_{-}\right)$are admissible for (1) on $R_{+}$and $R_{-}$respectively. As we show, the "bifurcation" condition which was trivial on $R_{+}$now plays a fundamental role in the development.

The next result examines the operator $L: \mathrm{E} \rightarrow \mathrm{B}$ where, as above, $L y(t)=$ $d y(t) / d t-A(t) y(t)$ and $\mathbf{B}, \mathbf{B}_{+}, \mathbf{B}_{-}, \mathbf{D}, \mathbf{D}_{+}, \mathbf{D}_{-}$and $\mathbf{E}$ are described in $\S \S 1$ and 2 respectively.

Each Banach space B employed in the sequel shall be tacitly assumed to satisfy the following condition. If $u$ is in B and $v$ is in $L$ with $|v(t)| \leqslant|u(t)|$ for almost all $t$ in $R$ then $v$ is in B and $|v|_{\mathrm{B}} \leqslant|u|_{\mathrm{B}}$.

THEOREM 5.1. Suppose that $\left(\mathbf{B}_{+}, \mathbf{D}_{+}\right)$and $\left(\mathbf{B}_{-}, \mathbf{D}_{-}\right)$are admissible for 
(1) on the domains $R_{+}$and $R_{-}$respectively. The operator $L$ is a closed linear operator whose nullity $\mathrm{N}(L)$ and range $\mathrm{R}(L)$ are closed subspaces of $\mathrm{E}$ and $\mathrm{B}$ respectively. These subspaces admit projection; hence, there exist projection operators $\Pi: \mathrm{E} \rightarrow \mathrm{E}$ and $\Omega: \mathrm{B} \rightarrow \mathrm{B}$ such that $\mathrm{N}(L)=\mathrm{R}(\Pi)$ and $\mathrm{R}(L)=\mathrm{N}(\Omega)$. Finally, $L$ has a bounded right inverse $K: \mathrm{B} \rightarrow \mathrm{E}$ with $L K=I_{\mathrm{R}(L)}$.

Proof. The $R$-analogue of Lemma $2.1[18, \mathrm{p} .289]$ as stated in the proof of Theorem 3.1 implies that $L$ is a closed operator.

Noting the definitions of the projections $P_{i \mathrm{D}}$, we have

$$
\mathbf{N}(L)=\left\{\phi: \phi \in \mathrm{E}, \phi(\cdot)=Y(t) \xi,\left(P_{-1 \mathrm{D}}+P_{1 \mathrm{D}}+P_{\infty \mathrm{D}}\right) \xi=0\right\} .
$$

Thus, $\mathbf{N}(L)$ is a finite-dimensional subspace of $\mathbf{E}$ and, as such, is closed and admits projection. Denote by $\Pi: \mathbf{E} \rightarrow \mathbf{E}$ the projection with $\mathbf{N}(L)=\mathbf{R}(I)$.

We show that $\mathbf{R}(L)$ has finite codimension in B and, therefore, is closed and admits projection. A recent result [13, Theorem 2] states that the problem $L: \mathrm{E} \rightarrow \mathrm{B}$ given by $L \phi=b$ has a solution $\phi^{*}$ valid on $R$ if and only if $b$ satisfies

$$
P_{\infty \mathrm{D}}\left\{T_{+}\left[b_{+}\right](0)-T_{-}\left[b_{-}\right](0)\right\}=0 .
$$

In (10), $T_{+}$and $T_{-}$are as defined in $\S 2$. Equation (10) is a finite set of "orthogonality" conditions and shows that $\mathbf{R}(L)$ has finite codimension. Thus, $\mathbf{R}(L)$ admits projection; let $\Omega$ designate a projection with $\mathbf{N}(\Omega)=\mathbf{R}(L)$.

An application of the closed graph theorem yields the existence of a bounded right inverse $K$ of $L$; see [21, p. 72]. This completes the proof of Theorem 5.1.

Following the procedure employed in $\S 3$, representations are given for the projections $\Pi$ and $\Omega$ and the inverse operator $K$ for a special case of the Banach spaces $\mathbf{B}$ and $\mathbf{D}$.

Consider the space $\mathbf{M}\left[R, \mathbf{R}^{n}\right]$ of all (equivalence classes of) locally integrable functions $f$ from $R$ to $\mathbf{R}^{n}$ with the property that

$$
\sup _{t \in R} \int_{t}^{t+1}|f(s)| d s<\infty .
$$

$\mathbf{M}\left[R, \mathbf{R}^{n}\right]$ is a Banach space with respect to the norm

$$
|f|_{M}=\sup _{t \in R} \int_{t}^{t+1}|f(s)| d s .
$$

In the next illustration, we utilize the hypothesis

(H.5). Let there exist positive constants $K_{j}, \alpha_{j}$ and supplementary projections $P_{j \mathrm{~L}}^{\infty}, j=0, \pm 1, \infty$ such that

$$
\left|Y(t) P_{0 \mathrm{~L}}^{\infty} Y^{-1}(s)\right| \leqslant K_{0} e^{-\alpha_{0}|t-s|}, \quad t, s \in R,
$$




$$
\begin{aligned}
& \left|Y(t) P_{\infty \mathrm{L}}^{\infty} Y^{-1}(s)\right| \leqslant \begin{cases}K_{\infty} e^{-\alpha_{\infty}(s-t)}, & 0 \leqslant t \leqslant s, \\
K_{\infty} e^{-\alpha_{\infty}(t-s)}, & t \geqslant s \geqslant 0,\end{cases} \\
& \left|Y(t)_{-1 \mathrm{~L}} Y^{-1}(s)\right| \leqslant \begin{cases}K_{-1} e^{-\alpha_{-1}(t-s)}, & t \geqslant s \geqslant 0, \\
K_{-1} e^{-\alpha_{-1}(t-s)}, & s \leqslant t \leqslant 0,\end{cases} \\
& \left|Y(t) P_{1 \mathrm{~L}} Y^{-1}(s)\right| \leqslant \begin{cases}K_{1} e^{-\alpha_{1}(t-s)}, & t \leqslant s \leqslant 0, \\
K_{1} e^{-\alpha_{1}(s-t)}, & 0 \leqslant t \leqslant s .\end{cases}
\end{aligned}
$$

The hypothesis (H.S) is necessary and sufficient for each of the pair of Banach spaces $\left(\mathrm{M}\left[R_{+}, \mathrm{R}^{n}\right], \mathrm{L}^{\infty}\left[R_{+}, \mathrm{R}^{n}\right]\right)$ and $\left(\mathrm{M}\left[R_{-}, \mathrm{R}^{n}\right], \mathrm{L}^{\infty}\left[R_{-}, \mathrm{R}^{n}\right]\right)$ to be admissible for (1) on $R_{+}$and $R_{-}$respectively (Coppel [6], Lovelady [17]).

THEOREM 5.2. Suppose that condition (H.5) is satisfied.

(i) Let $\Phi(\cdot)$ denote the $n \times r$ matrix-valued function whose columns form a basis for $\operatorname{sp}\left\{Y(\cdot) P_{0 L^{\infty}}\right\}$. Then, the $r \times r$ matrix $C \equiv \int_{-\infty}^{\infty} \Phi^{T}(s) \Phi(s) d s$ is well defined and nonsingular provided $P_{\mathrm{OL}^{\infty}} \neq 0$. Furthermore, the map $\Pi: \mathrm{E}\left(\mathrm{L}^{\infty}, \mathrm{M}\right)$ $\rightarrow \mathrm{E}\left(\mathrm{L}^{\infty}, \mathrm{M}\right)$ given by

$$
\Pi \phi=\Phi(\cdot) C^{-1} \int_{-\infty}^{\infty} \Phi^{T}(s) \phi(s) d s
$$

is a projection of $\mathrm{E}\left(\mathrm{L}^{\infty}, \mathrm{M}\right)$ into $\mathrm{E}\left(\mathrm{L}^{\infty}, M\right)$ with $\mathrm{N}(L)=\mathbf{R}(\mathrm{II})$.

(ii) Let $\Psi(\cdot)$ denote the $k \times n$ matrix whose rows form a basis for $\operatorname{sp}\left\{P{ }_{\infty}^{\infty} Y^{\infty} Y^{-1}(\cdot)\right\}$. Then, the matrix $D \equiv \int_{-\infty}^{\infty} \Psi(s) \Psi^{T}(s) d s$ is well defined and nonsingular provided $P_{\infty L^{\infty}} \neq 0$. Furthermore, the map $\Omega: M\left[R, R^{n}\right] \rightarrow$ $\mathrm{M}\left[R, \mathrm{R}^{n}\right]$ given by

$$
\Omega \phi=\Psi^{T}(\cdot) D^{-1} \int_{-\infty}^{\infty} \Psi(s) \phi(s) d s
$$

is a projection of $M\left[R, R^{n}\right]$ into itself with $R(L)=(I-\Omega) M\left[R, R^{n}\right]$.

(iii) $A$ bounded right inverse $K: M\left[R, \mathrm{R}^{n}\right] \rightarrow E\left(\mathrm{~L}^{\infty}, \mathrm{M}\right)$ for $L: \mathrm{E}\left(\mathrm{L}^{\infty}, \mathrm{M}\right)$ $\rightarrow \mathrm{M}\left[R, \mathrm{R}^{n}\right]$ is given by $K=T(I-\Omega)$ where $T$ is defined on $\mathrm{R}(L)$ by

$$
\begin{aligned}
(T b)(t)= & \int_{-\infty}^{t} Y(t) P_{-1 \mathrm{~L}}^{\infty} Y^{-1}(s) b(s) d s+\int_{0}^{t} Y(t) P_{0 \mathrm{~L}} Y^{-1}(s) b(s) d s \\
& -\int_{t}^{\infty} Y(t)\left(P_{1 \mathrm{~L}}{ }^{\infty}+P_{\infty \mathrm{L}}\right) Y^{-1}(s) b(s) d s, \quad t \in R_{+}:
\end{aligned}
$$

and for $t$ in $R_{-}$, 


$$
\begin{aligned}
(T b)(t)= & \int_{0}^{t} Y(t) P_{0 \mathrm{~L}} Y^{-1}(s) b(s) d s-\int_{t}^{\infty} Y(t) P_{1 \mathrm{~L}} Y^{-1}(s) b(s) d s \\
& +\int_{-\infty}^{t} Y(t)\left(P_{-1 \mathrm{~L}}^{\infty}+P_{\infty}{ }^{\infty}\right) Y^{-1}(s) b(s) d s .
\end{aligned}
$$

Proof. The verification of conclusion (i) is similar to that of Theorem 3.2, part (i); hence, the details are omitted.

To establish (ii), it follows from [13] that given $f$ in $M\left[R, \mathbf{R}^{n}\right]$, there exists a $y$ in $\mathrm{E}\left(L^{\infty}, M\right)$ with $L y .=f$ if and only if $\int_{-\infty}^{\infty} P P_{\infty} L^{\infty} Y^{-1}(s) f(s) d s=0$. However, $\int_{-\infty}^{\infty} P{ }_{\infty}^{\infty} Y^{-1}(s) f(s) d s=0$ if and only if $\Omega f=0$; that is, $f$ is in $(I-\Omega) M$. It follows that $\mathbf{R}(L)=(I-\Omega) \mathbf{M}$.

A direct computation shows that $L T b=b$ for all $b$ in $\mathbf{R}(L)$, that is, $b$ in $\mathbf{M}$ with $\Omega b=0$. Hypothesis (H.5) implies that $T$ maps $\mathbf{R}(L)$ into $\mathbf{M}$. The operator $K=T(I-\Omega)$ extends $T$ to all of $\mathrm{M}$.

6. Abstract formulation of the nonlinear problem on $R$. The development of the first portion of this section will be much like that of $\S 4$. Notation used are the $R$-analogues of those in $\S 4$.

The fundamental results and notation from $\$ 5$ on the linear operator $L$ : $\mathbf{E} \rightarrow \mathbf{B}$ are also utilized in the sequel.

THEOREM 6.1. Suppose that $\left(\mathbf{B}_{+}, \mathbf{D}_{+}\right)$and $\left(\mathbf{B}_{-}, \mathbf{D}_{-}\right)$are admissible for (1) on the domains $\mathbf{R}_{+}$and $\mathbf{R}_{-}$respectively. Let $N: \mathbf{E} \times \Lambda \rightarrow B$ be a weak nonlinearity. Suppose that $M=|\Phi(\cdot)|_{\mathrm{D}}$ where $\Phi(\cdot)$ denotes the $n \times p$ matrix function whose columns form a basis for $\operatorname{sp}\left\{Y(\cdot) P_{\mathrm{OD}}\right\}$. Then, corresponding to each $\rho>0$, there exists $a \sigma=\sigma(\rho)>0$ such that the perturbation problem

$$
L \phi=N(\phi, \lambda), \quad \Omega N(\phi, \lambda)=0, \quad \Pi \phi=\Phi(\cdot) a_{0}, \quad \phi \in \mathrm{S}_{\rho}(\mathrm{E}),
$$

has a unique solution $\phi^{*}\left(\cdot, a_{0}, \lambda\right)$ for each $\left(a_{0}, \lambda\right) \in \mathbf{S}_{\rho / 2 M}\left(\mathbf{R}^{p}\right) \times \mathbf{S}_{\sigma}(\Lambda)$. Furthermore, $\phi^{*}\left(\cdot, a_{0}, \lambda\right)$ is continuous as a map from $S_{\rho / 2 M}\left(R^{p}\right) \times S_{\sigma}(\Lambda)$ into $\mathbf{E}$ and satisfies $\lim _{\lambda \rightarrow 0} \phi^{*}\left(\cdot, a_{0}, \lambda\right)=\Phi(\cdot) a_{0}$ for each $a_{0}$ in $\mathbf{S}_{\rho / 2 M}\left(\mathbf{R}^{p}\right)$.

Proof. The problem (11) may be written as

$$
\phi=(I-\Pi) K(I-\Omega) N(\phi, \lambda)+\Phi(\cdot) a_{0} .
$$

A solution of this equation can be obtained by establishing the existence of a fixed point of the operator $T: \mathbf{E} \times \mathbf{R}^{p} \times \Lambda \rightarrow \mathbf{E}$ defined as

$$
T\left(\phi, a_{0}, \lambda\right)=(I-\Pi) K(I-\Omega) N(\phi, \lambda)+\Phi(\cdot) a_{0} .
$$

Employing the bounds on $\Pi, \Omega$, and $K$, it can be shown that $T\left(\cdot, a_{0}, \lambda\right)$ is uniformly contracting on the set $\left\{\phi: \phi \in S_{\rho}(E), \Pi \phi \in S_{\rho / 2}(E)\right\}$ with respect to the parameter set $S_{\rho / 2 M}\left(R^{p}\right) \times S_{\sigma}(\Lambda)$ provided $\sigma$ is sufficiently small. The uniform 
contraction principle yields a unique fixed point of $T$ with the desired properties. This completes an indication of the proof of Theorem 6.1.

REMARK 6.1. If $N$ is not differentiable then the Schauder-Tychonoff fixed point can be employed in this setting; see [15] and $\S 4$.

The next result is a modification of an interesting bifurcation result of $S$. Bancroft [1]. Our setting is distinct from that of [1] where it is required that the linear operator maps a Banach space into itself while the range of the linear operator employed here need not be the same as the domain; however, the details of the proofs are similar. The work of Bancroft on periodic solutions shows that, in general, it is not the case that the solution $\phi(\cdot, \lambda)$ of $(11)$ is continuous at $\lambda=0$. The next result is concerned with the determination of an appropriate asymptotic vector $a_{0}$ which indicates the solution of the linear system that is obtained in the limit as $\lambda \rightarrow 0$.

Let $\Gamma$ denote a compact set on the surface of the unit sphere in $\Lambda$. Define $\mathbf{C}(\Gamma, \sigma)=\{\lambda \in \Lambda:|\lambda| \leqslant \sigma, \lambda /|\lambda| \in \Gamma$ if $\lambda \neq 0\}$, and $\mathbf{C}^{\prime}(\Gamma, \sigma)=\mathbf{C}(\Gamma, \sigma)-\{0\}$. A space complementary to $\mathbf{N}(\Omega)=\mathbf{R}(L)$ will be denoted by $\mathbf{R}_{c}(L)$.

THEOREM 6.2. Let the hypothesis of Theorem 6.1 be satisfied and suppose that the map $H: \mathbf{R}^{p} \times \Lambda \rightarrow \mathbf{R}_{c}(L)$ is defined by

$$
H\left(a_{0}, \lambda\right)=\Omega N_{\lambda}\left(\Phi(\cdot) a_{0}, 0\right) \lambda .
$$

Suppose that there exists a continuous map $\alpha: \Gamma \rightarrow \mathbf{R}^{p}$ with the properties that

$$
H(\alpha(\lambda), \lambda)=0, \quad \operatorname{det}\left|\partial H(\alpha(\lambda), \lambda) / \partial a_{0}\right| \neq 0
$$

for all $\lambda \in \Gamma$. Then, there exists $a \sigma=\sigma(\Gamma)>0$ such that the problem

$$
L \phi=N(\phi, \lambda), \quad \Omega N(\phi, \lambda)=0
$$

has a solution $\phi^{*}(\cdot, \lambda): \mathbf{C}(\Gamma, \sigma) \rightarrow \mathrm{D}$ for which $\phi^{*}(\cdot, \lambda)$ is uniformly bounded on $\mathrm{C}(\Gamma, \sigma)$ and continuous on $\mathrm{C}^{\prime}(\Gamma, \sigma)$. Furthermore, $\phi^{*}=\phi^{*}(\cdot, \lambda)$ has the representation

$$
\phi^{*}(\cdot, \lambda)=\Phi(\cdot) \alpha(\lambda|\lambda|)+\nu(\cdot, \lambda)
$$

where $\nu(\cdot, \lambda): \mathbf{C}^{\prime}(\Gamma, \sigma) \rightarrow \mathrm{D}$ satisfies $\lim _{\lambda \rightarrow 0} \nu(\cdot, \lambda)=0$.

Proof. For $\lambda \in \Gamma$, let $\alpha(\lambda)$ satisfy $|\alpha(\lambda)| \leqslant b_{1}<\rho / 2 M$. Select $\epsilon_{0}$ such that $b_{1}+\epsilon_{0}<\rho / 2 M$. Define the map $F: \mathbf{S}_{\epsilon_{0}}\left(\mathbf{R}^{p}\right) \times \mathbf{C}^{\prime}(\Gamma, \sigma) \rightarrow \mathbf{R}_{c}(L)$ by

$$
F(\omega, \lambda)=\Omega N(\Phi(\cdot)[\alpha(\lambda|\lambda|)+\omega], \lambda) .
$$

The existence of a $\sigma=\sigma(\Gamma)>0$ and a continuous map $\omega: \mathbf{C}^{\prime}(\Gamma, \sigma) \rightarrow \mathbf{S}_{\epsilon_{0}}\left(\mathbf{R}^{p}\right)$, such that $F(\omega(\lambda), \lambda)=0$ and $\lim _{\lambda \rightarrow 0} \omega(\lambda)=0$, will be demonstrated. 
First, we observe that $\partial F(0, \lambda) / \partial \omega$ is invertible for all $\lambda$ in $C^{\prime}(\Gamma, \sigma)$ if $\sigma$ is small. This is a consequence of the relationship

$$
\begin{aligned}
\partial F(0, \lambda) / \partial \omega & =\Omega \frac{\partial}{\partial \omega}\left[N_{\lambda}\left(\Phi(\cdot) \alpha\left(\frac{\lambda}{|\lambda|}\right)+\omega, \lambda\right)+o(\lambda)\right](0, \lambda) \\
& =|\lambda|\left\{\partial H(\alpha(\lambda /|\lambda|), \lambda /|\lambda|) / \partial a_{0}+o(\lambda /|\lambda|)\right\} \text { as } \lambda \rightarrow 0,
\end{aligned}
$$

and the hypothesis that $\partial H(\alpha(\lambda), \lambda) / \partial a_{0}$ exists for each $\lambda$ in $\Gamma$ provided $\sigma$ is sufficiently small.

Expanding $F$ in a Taylor series about $\omega=0$, we have

$$
F(\omega, \lambda)=F(0, \lambda)+\frac{\partial F}{\partial \omega}(0, \lambda) \omega+P(\omega, \lambda)
$$

where the remainder $P$ is defined by (13). In order that the bifurcation equation $\Omega N=0$ hold, it is required that for each $\lambda, \omega=\omega(\lambda)$ be chosen so that $F(\omega, \lambda)$ $=0$. To obtain the desired asymptotic behavior, we also need $\lim _{\lambda \rightarrow 0} \omega(\lambda)=0$. These two properties will be established through consideration of the operator $J$ : $S_{\epsilon_{0}}\left(R^{p}\right) \times C^{\prime}(\Gamma, \sigma) \rightarrow \mathrm{N}(L)$ defined by

$$
J(\omega, \lambda)=-\frac{\partial F}{\partial \omega}(0, \lambda)^{-1}\{P(\omega, \lambda)+F(0, \lambda)\} .
$$

We note that $\omega(\lambda)$ satisfies $F(\omega, \lambda)=0$ if and only if $\omega(\lambda)$ is a fixed point of $J(\cdot, \lambda)$. To find a fixed point $\omega(\lambda)$ of $J(\cdot, \lambda)$ that also satisfies the asymptotic condition $\lim _{\lambda \rightarrow 0} \omega(\lambda)=0$, the uniform contraction principle is employed.

Estimates, to be used to show that $J$ is a contraction, for the individual functions comprising $J$ are now found. We note that the hypothesis $H(\alpha(\lambda /|\lambda|), \lambda)=0$ for $\lambda$ in $C^{\prime}(\Gamma, \sigma)$ implies

$$
F(0, \lambda)=\Omega[N(\Phi(\cdot) \alpha(\lambda /|\lambda|), \lambda)-N(\Phi(\cdot) \alpha(\lambda /|\lambda|), 0)] .
$$

An application of the mean value theorem leads to the existence of a continuous function $\psi_{1}=\psi_{1}(\sigma)$ with $\psi_{1}(0)=0$ such that

$$
|F(0, \lambda)| \leqslant \psi_{1}(\sigma)|\lambda|, \lambda \in S_{\sigma}(\Lambda) .
$$

It follows from (12) that whenever $\sigma$ is sufficiently small,

$$
\left.\left|[\partial F(0, \lambda) / \partial \omega]^{-1} \leqslant\right| \lambda\right|^{-1} \mu
$$

for some positive constant $\mu$.

Finally, from (13), (12) and some estimations using the mean value theorem, we find a continuous function $\psi_{2}=\psi_{2}(\epsilon)$ with $\psi_{2}(0)=0$ such that

$$
\left|P\left(\omega_{1}, \lambda\right)-P\left(\omega_{2}, \lambda\right)\right| \leqslant|\lambda| \psi_{2}(\epsilon)\left|\omega_{1}-\omega_{2}\right|
$$

for all $\omega_{1}, \omega_{2}$ in $S_{\epsilon}\left(R^{p}\right)$. 
Combining the above inequalities, we obtain a continuous function $\epsilon=\epsilon(\sigma)$ with $\lim _{\sigma \rightarrow 0^{+}} \epsilon(\sigma)=0$ such that $J(\cdot, \lambda)$ is uniformly contracting on $S_{\epsilon(\sigma)}\left(R^{p}\right)$ for all $\lambda \stackrel{\sigma \rightarrow}{\text { in }}$ the parameter set $C^{\prime}(\Gamma, \sigma)$. Let $\omega(\cdot, \lambda)$ denote the fixed point of $J(\cdot, \lambda)$ with asymptotic property $\lim _{\lambda \rightarrow 0} \omega(\cdot, \lambda)=0$.

The initial position $a_{0}=a_{0}(\cdot, \lambda)=\alpha(\lambda /|\lambda|)+\omega(\cdot, \lambda)$ will be utilized in conjunction with the conclusion of Theorem 6.1. Consider the solution $\phi^{*}=$ $\phi^{*}\left(\cdot, a_{0}(\cdot, \lambda), \lambda\right)$ of (11); Theorem 6.1 implies that there exists a function $\omega^{*}(\cdot, \lambda)$, defined on $C^{\prime}(\Gamma, \sigma)$ with $\lim _{\lambda \rightarrow 0} \omega^{*}(\cdot, \lambda)$ and $\phi^{*}\left(\cdot, a_{0}(\cdot, \lambda), \lambda\right)-$ $\Phi(\cdot) a_{0}(\cdot, \lambda)=\omega^{*}(\cdot, \lambda)$. From this representation we obtain

$$
\phi^{*}\left(\cdot, a_{0}(\cdot, \lambda), \lambda\right)-\Phi(\cdot) \alpha(\lambda /|\lambda|)=\nu(\cdot, \lambda)
$$

where $v(\cdot, \lambda) \equiv \omega^{*}(\cdot, \lambda)-\Phi(\cdot) \omega(\cdot, \lambda)$ clearly satisfies $\lim _{\lambda \rightarrow 0} \nu(\cdot, \lambda)=0$. This concludes the proof of Theorem 6.2.

As an illustration of Theorem 6.2, the following is given.

THEOREM 6.3. Let the hypothesis of Theorem 5.2 be satisfied. Let $g$ : $\boldsymbol{R} \times \mathbf{R}^{\boldsymbol{n}} \times \boldsymbol{\Lambda} \rightarrow \mathbf{R}^{\boldsymbol{n}}$ be continuous together with its second partial derivatives and suppose that $g(t, x, 0)=0$ for $(t, x)$ in $R \times R^{n}$. Define

$$
H\left(a_{0}, \lambda\right)=\int_{-\infty}^{\infty} \Psi(t) \frac{\partial g}{\partial \lambda}\left(t, \Phi(t) a_{0}, 0\right) \lambda d t .
$$

Suppose that a continuous map $\alpha: \Gamma \rightarrow \mathbf{R}^{p}$ can be found such that $H(\alpha(\lambda), \lambda)$ $=0$ and $\operatorname{rank}\left(\partial H / \partial a_{0}\right)(\alpha(\lambda), \lambda)=k$ for $\lambda \in \Gamma$. Then, there exists a positive constant $\sigma=\sigma(\Gamma)$ such that $x^{\prime}=A(t) x+g(t, x, \lambda)$ has an $L^{\infty}(R)$-solution $x^{*}=$ $x^{*}(\cdot, \lambda)$ that is uniformly bounded on $\mathrm{C}(\Gamma, \sigma)$, continuous on $\mathrm{C}^{\prime}(\Gamma, \sigma)$ and satisfies

$$
x^{*}(\cdot, \lambda)=\Phi(\cdot) \alpha(\lambda /|\lambda|)+\nu(\cdot, \lambda)
$$

where $\lim _{\lambda \rightarrow 0} \nu(\cdot, \lambda)=0$.

Acknowledgment. The author would like to thank Professor Stephen Bancroft for several helpful correspondences concerning this article. He also kindly provided a preliminary version of reference [1].

\section{REFERENCES}

1. Stephen Bancroft, Perturbations with several independent parameters, J. Math. Anal. Appl. 50 (1975), 384-414.

2. S. Bancroft and T. G. Hallam, Bounded solutions of nonlinear differential equations with parameters, SIAM J. Math. Anal. 6 (1975), 236-241.

3. T. F. Bridgland, Jr., On the boundedness and uniform boundedness of solutions of nonhomogeneous systems, J. Math. Anal. Appl. 12 (1965), 471-487. MR 32 \# 7846.

4. L. Cesari, Asymptotic behavior and stability problems in ordinary differential equations, Ergebnisse der Mathematik und ihrer Grenzgebiete, N. F., Heft 16, Springer-Verlag, Berlin; Academic Press, New York, 1963. MR 27 \# 1661. 
5. R. Conti, On the boundedness of solutions of ordinary differential equations, Funkcial. Ekvac. 9 (1966), 23-26. MR 37 \# 3102.

6. W. A. Coppel, On the stability of ordinary differential equations, J. London Math. Soc. 39 (1964), 255-260. MR 29 \# 1393.

7. - Dichotomies and stability theory, Sympos. on Differential Equations and Dynamical Systems, Lecture Notes in Math., vol. 206, Springer-Verlag, New York, 1971, pp. 160-162.

8. - Stability and asymptotic behavior of differential equations, Heath, Boston, Mass., 1965. MR 32 \# 7875.

9. C. Corduneanu, Sur certaines systèmes différentielles nonlinéaires, An. Şti. Univ “Al. I. Cuza” Iaşi Seç̧. I. 6 (1960), 257-260. MR 23 \# A1906.

10. J. K. Hale, Oscillations in nonlinear systems, McGraw-Hill, New York, 1963. MR 27 \# 401.

11. - Ordinary differential equations, Wiley, New York, 1969.

12. T. G. Hallam, On asymptotic equivalence of the bounded solutions of two systems of differential equations, Michigan Math. J. 16 (1969), 353-363. MR 40 \# 5983.

13. T. G. Hallam and D. L. Lovelady, On admissibility for differential equations on $R$, J. London Math. Soc. (2) 11 (1975), 49-52.

14. P. Hartman, Ordinary differential equations, Wiley, New York, 1964. MR 30 \# 1270.

15. P. Hartman and N. Onuchic, On the asymptotic integration of ordinary differential equations, Pacific J. Math. 13 (1963), 1193-1207. MR 28 \# 293.

16. G. Ladas and V. Lakshmikantham, Differential equations in abstract spaces, Academic Press, New York, 1972.

17. D. L. Lovelady, Admissibility criteria for Stepanoff function spaces (to appear).

18. J. L. Massera and J. J. Schäffer, Linear differential equations and functional analysis. IV, Math. Ann. 139 (1960), 287-342. MR 22 \# 8181.

19. - Linear differential equations and function spaces, Pure and Appl. Math., vol. 21, Academic Press, New York, 1966. MR 35 \# 3197.

20. N. Minorsky, Nonlinear oscillations, Van Nostrand, Princeton, N. J., 1962. MR 25 \# 1339.

21. L. Nirenberg, Functional analysis, Lecture Notes, New York University, 1960.

22. H. L. Royden, Real analysis, 2nd ed., Macmillian, New York, 1968.

DEPARTMENT OF MATHEMATICS, FLORIDA STATE UNIVERSITY, TALLAHASSEE, FLORIDA 32306

Current address: Department of Mathematics, University of Georgia, Athens, Georgia 